\title{
Tactile and Ultrasound Image Fusion for Functional Assessment of the Female Pelvic Floor
}

\author{
Vladimir Egorov ${ }^{1}$, Heather van Raalte ${ }^{2}$, S. Abbas Shobeiri ${ }^{3}$ \\ ${ }^{1}$ Advanced Tactile Imaging, Trenton, NJ, USA \\ ${ }^{2}$ Princeton Urogynecology, Princeton, NJ, USA \\ ${ }^{3}$ INOVA Fairfax Hospital, Falls Church, VA, USA \\ Email: egorov@tactile-imaging.com
}

How to cite this paper: Egorov, V., van Raalte, H. and Shobeiri, S.A. (2021) Tactile and Ultrasound Image Fusion for Functional Assessment of the Female Pelvic Floor. Open Journal of Obstetrics and Gynecology, 11, 674-688.

https://doi.org/10.4236/ojog.2021.116063

Received: May 4, 2021

Accepted: June 8, 2021

Published: June 11, 2021

Copyright $\odot 2021$ by author(s) and Scientific Research Publishing Inc. This work is licensed under the Creative Commons Attribution International License (CC BY 4.0).

http://creativecommons.org/licenses/by/4.0/

\begin{abstract}
Introduction: The true etiology of pelvic organ prolapse and urinary incontinence and variations observed among individuals are not entirely understood. Tactile (stress) and ultrasound (anatomy, strain) image fusion may furnish new insights into the female pelvic floor conditions. This study aimed to explore imaging performance and clinical value of vaginal tactile and ultrasound image fusion for characterization of the female pelvic floor. Methods: A novel probe with 96 tactile and 192 ultrasound transducers was designed. Women scheduled for a urogynecological visit were considered eligible for enrollment to observational study. Intravaginal tactile and ultrasound images were acquired for vaginal wall deformations at probe insertion, elevation, rotation, Valsalva maneuver, voluntary contractions, involuntary relaxation, and reflex pelvic muscle contractions. Biomechanical mapping has included tactile/ultrasound imaging and functional imaging. Results: Twenty women were successfully studied with the probe. Tactile and ultrasound images for tissues deformation as well as functional images were recorded. Tactile (stress) and ultrasound (strain) images allowed creation of stress-strain maps for the tissues of interest in absolute scale. Functional images allowed identification of active pelvic structures and their biomechanical characterization (anatomical measurements, contractive mobility and strength). Fusion of the modalities has allowed recognition and characterization of levator ani muscles (pubococcygeal, puborectal, iliococcygeal), perineum, urethral and anorectal complexes critical in prolapse and/or incontinence development. Conclusions: Vaginal tactile and ultrasound image fusion provides unique data for biomechanical characterization of the female pelvic floor. Bringing novel biomechanical characterization for critical soft tissues/structures may provide extended scientific knowledge and improve clinical practice.
\end{abstract}




\section{Keywords}

Tissue Elasticity, Pelvic Support, Pelvic Function, Ultrasound, Tactile, Biomechanical Mapping

\section{Introduction}

Pelvic organ prolapse (POP) is a highly prevalent condition affecting $40-50 \%$ of women in the U.S. during their lifetime [1] [2]. Urinary incontinence (UI) affects up to $48 \%$ of women [3]. The true etiology of POP and UI and variations observed among individuals are not entirely understood. These disorders are thought to share common pathogeneses, weakening (elasticity changes) of the connective support tissues and pelvic floor muscle dysfunction [4] [5] [6].

The lifetime surgery risk for either UI or POP in women is about 20\% [7] [8]. A representative study reported a POP surgical failure rate of $61.5 \%$ for uterosacral ligament suspension and $70.3 \%$ for sacrospinous ligament fixation groups [9]. Substantial improvement is possible if patients with the diseased pelvic floor conditions undergo imaging and biomechanical diagnostic tests, the results of which could be fed into a patient-specific optimal treatment [10] [11] [12].

Conventional ultrasound is available for the imaging of pelvic floor organs. Perineal or translabial, transvaginal, and abdominal ultrasounds are increasingly used for assessing UI and POP. Ultrasound, as a part of the diagnostic workup, enables morphological and dynamic assessment of the female pelvic floor [13] [14] [15].

Biofeedback with vaginal pressure measurements (air balloon or 1 - 2 pressure sensors) has been widely utilized in the treatment of pelvic floor dysfunctions, mainly by promoting patient knowledge about muscle contraction. Urodynamics allows pressure measurements in the bladder, urethra, vagina, and rectum with catheters. A Vaginal Tactile Imager provides a high-resolution mapping of pressures and assesses the strength of pelvic floor muscles within the vagina to assist in diagnosis [16].

None of the above-listed techniques allows biomechanical mapping (stress-strain or functional) of the female pelvic floor structures with accurate anatomical identification.

Elastography, or elasticity imaging, involves the application of stress to soft tissue and measurement of the resulting tissue mechanical response. There are several methods available to apply stress to a tissue and measure the resulting response. The stress can be generated from an external source such as a compression probe, an external vibrator, acoustic radiation force, or physiological sources of motion (cardiac motion and fluid flow). The most common measurement methods of tissue response (strain) include ultrasound, magnetic resonance imaging, and mechanical/tactile imaging [17]. The strain ultrasound does not allow soft tissue characterization on an absolute scale without stress 
data. Shear wave elastography seems too complicated for intravaginal application. The MRI elastography is a complex technology with a relatively high cost. The tactile imaging probe translates stress data from the surface of deformed soft tissue into a 2D/3D image, allowing the assessment of tissue elasticity, heterogeneity, and extraction of the non-linear elasticity component. Tactile imaging is a relatively simple and non-expensive technique, but requires in-depth anatomical strain data for accurate tissue characterization.

The approach reported in this article is intended to overcome the basic limitations of the strain ultrasound imaging (no stress data) and tactile imaging (no anatomy in depth) now being applied for the female pelvic floor. The objectives of this study were to explore imaging performance, clinical value and safety of vaginal tactile and ultrasound image fusion in a pilot study. Additionally, there will be an exploration of a new possibility arising from that fusion-a functional imaging of the pelvic floor structure/muscles contributing to pelvic floor disorders.

\section{Materials and Methods}

\subsection{Vaginal Tactile Ultrasound Imager}

The Vaginal Tactile Ultrasound Imager (TIUSv) includes a probe, ultrasound electronics, proprietary tactile data acquisition/calibration electronics, and a touchscreen computer. The probe comprises two tactile arrays (48 sensors per probe side), an ultrasound transducer array of 192 elements, two temperature sensors, a micro-heater, and a 3D orientation sensor (see Figure 1). The tactile sensor (capacitor) includes 1) a ferroelectret film used as a dielectric, and 2) flexible pads used as electro-conductive plates. Sensor step along the probe is 2.5 $\mathrm{mm}$. Sensor array temperature is maintained at $35^{\circ} \mathrm{C} \pm 1.0^{\circ} \mathrm{C}$ to eliminate temperature dependence of sensor readings and for the patient's comfort. Three-axis accelerometer ADXL327 (Analog Devices, Norwood, MA, USA) is used as a probe orientation sensor.

The linear 192-element ultrasound array was fabricated by Vermon (Tours, France) according to our specifications. It has an operational length of $63.4 \mathrm{~mm}$, center frequency of $5.0 \mathrm{MHz}$, pitch of $0.33 \mathrm{~mm}$, and elevation aperture of 2.0 $\mathrm{mm}$. The tactile and ultrasound array are molded in the same silicone as used for the surface layer of the probe. Ultrasound electronics ArtUs (Telemed, Vilnius, Lithuania) was used in the device; it has 64 channels multiplexed to 192 transducers.

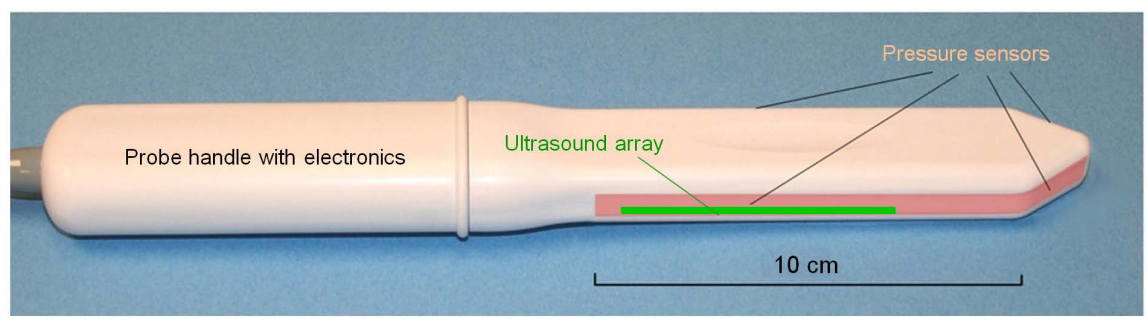

Figure 1. Vaginal probe with 96 tactile and 192 ultrasound transducers. 
Tactile data were synchronized with ultrasound data acquisition. Tactile and ultrasound images were visualized in real-time in two separate panels. We developed a stand-alone data processing application to visualize and analyze the recorded tactile and ultrasound data after the patient examination.

\subsection{Study Design}

The Western Institutional Review Board (IRB) issued approval No. 20193069 on December 26, 2019 for clinical protocol "Tactile and Ultrasound Imaging of the Female Pelvic Floor" for observational case-controlled study at Princeton Urogynecology (Princeton, NJ, USA) and INOVA Medical Group (Falls Church, VA, USA). A written informed consent was obtained from all enrolled subjects. This clinical research was conducted in compliance with the Health Insurance Portability and Accountability Act. The study objectives included 1) assessment of imaging performance and clinical value of the device, 2) identification of basic pelvic floor structures, and 3) exploration of normal versus POP conditions. Women scheduled for a urogynecological visit were considered eligible for the enrollment. The study inclusion criteria were 1) Age of 21 years or more, 2) no prior pelvic floor surgery, and 3) one of the following: normal pelvic floor conditions or POP stage I-III affecting one or more vaginal compartments. The study exclusion criteria were as follows: 1) prior pelvic surgery related to POP, incontinence or other reason, 2) active skin infection or ulceration within the vagina, 3) presence of a vaginal septum, 4) active cancer of the colon, rectum wall, cervix, vagina, uterus or bladder, 5) ongoing radiation therapy for pelvic cancer, 6) impacted stool, 7) severe hemorrhoids, 8) significant circulatory or cardiac conditions that could cause excessive risk from the examination as determined by a physician, 9) current pregnancy, and 10) cognitive impairment. The analyzed dataset of 20 subjects participated in the study was characterized by the following metrics: average patient age was 51.9 years (from 27 to 83 ); parity of 1.8 (from 0 to 4); 14 subjects had anterior POP, 10 had posterior POP, and 8 had uterine POP; 5 subjects had no POP, 3 had stage I POP, 8 had stage II POP, and 4 subjects had stage III POP; 8 subjects had stress urinary incontinence (SUI), 4 had UI and 3 subjects had an overactive bladder (OAB).

The TIUSv examination data were obtained and recorded during the scheduled urogynecological visits. The study workflow comprised of the following steps: 1) Recruiting women who had not had a pelvic surgery; 2) Acquisition of clinical diagnostic information related to the inclusion/exclusion criteria (age, POP stage, co-morbidities) by standard clinical means; 3) Subject enrollment; 4) Performing a TIUSv examination in lithotomic position; and 6) Analyzing TIUSv-recorded data and composing tactile ultrasound image fusion. Prior to the TIUSv examination, a standard physical examination was performed, including a bimanual pelvic examination and Pelvic Organ Prolapse Quantification (POP-Q) [18]. The pelvic floor conditions were categorized by the stage of the prolapse, based on the maximum stage from anterior, posterior, and uterine prolapse. For each of the subjects, the pelvic floor evaluation was performed by their urogy- 
necologists with the treatment plan prescribed independent of this study.

\subsection{Examination Procedure}

The TIUSv examination procedure consisted of eight Tests: 1) probe insertion, 2) elevation, 3) rotation, 4) Valsalva maneuver, 5) voluntary muscle contraction (anterior versus posterior compartment), 6) voluntary muscle contraction (left versus right side), 7) involuntary muscle relaxation, and 8) reflex muscle contraction (cough). The TIUSv probe allows $3-15 \mathrm{~mm}$ tissue deformation at the probe insertion (Test 1), $20-45 \mathrm{~mm}$ tissue deformation at the probe elevation (Test 2), $5-7 \mathrm{~mm}$ deformation at the probe rotation (Test 3), and recording of dynamic responses at pelvic muscle contractions (Tests 4 - 8). Tests 1 - 5 and 7 8 provide data for anterior/posterior compartments, while Test 6 provides data for left/right sides. The full TIUSv examination takes 3 - 5 minutes.

\section{Results}

In 20 cases, the enrolled women were successfully studied with the TIUSv; the tactile and ultrasound imaging data were recorded. Figure 2 presents an example
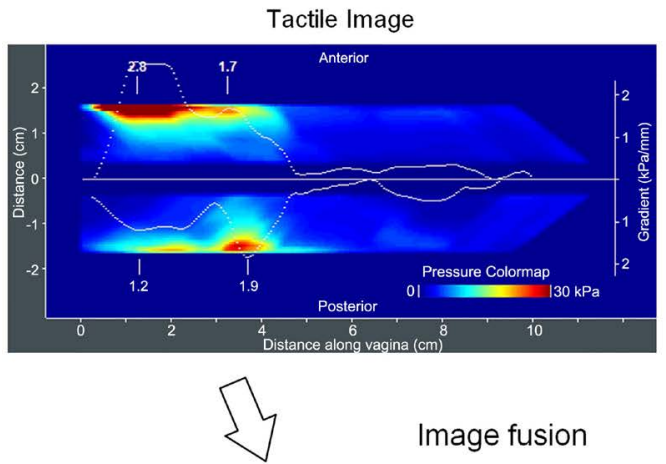

Image fusion
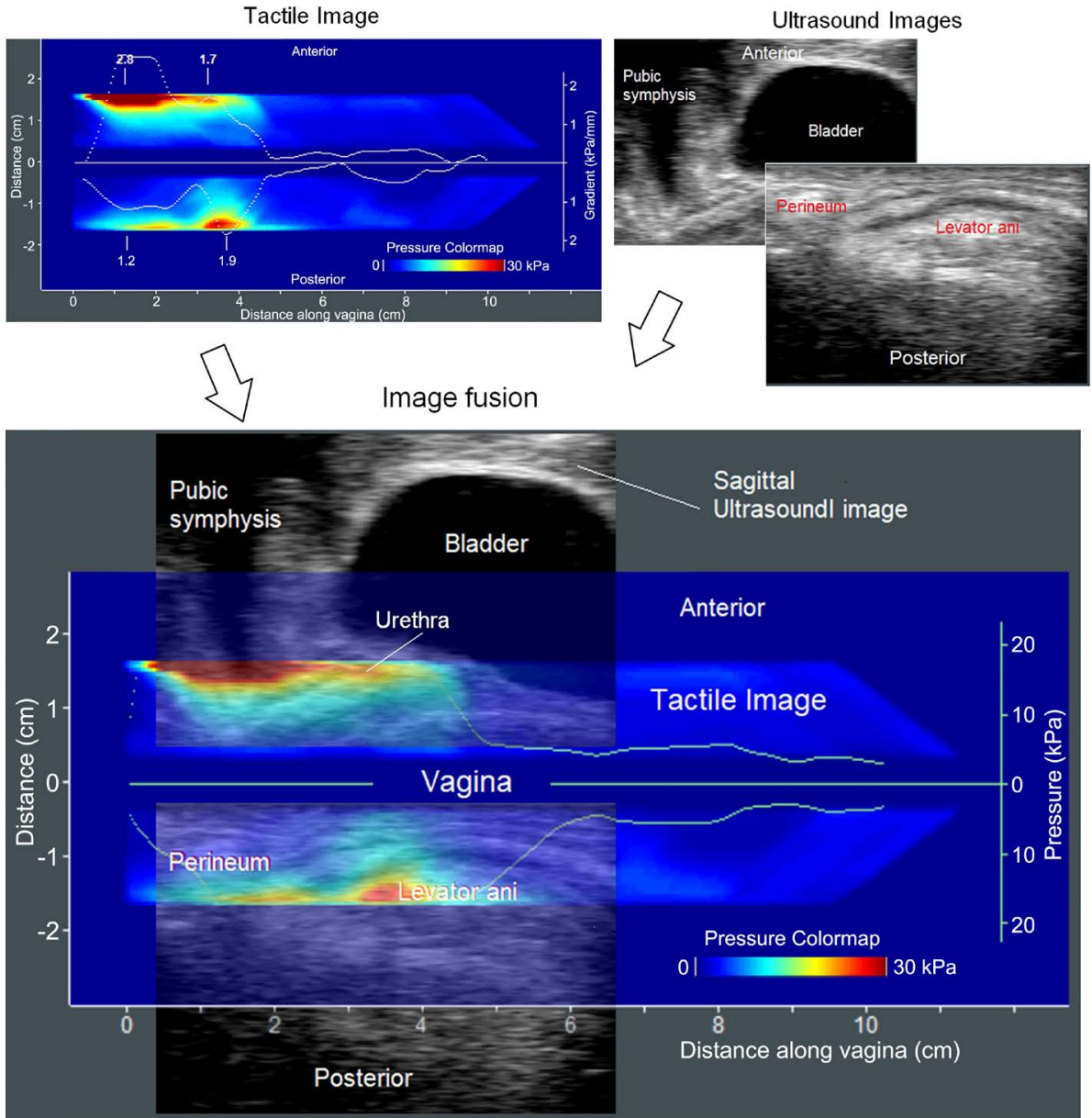

Figure 2. Tactile and Ultrasound imaging fusion for Test 1 (probe insertion) allows elasticity assessment for critical structures; 34 y.o. women with normal pelvic floor conditions. 
of tactile ultrasound image fusion for Test 1 (probe insertion). It allows identification of specific pelvic structure contributing to the tactile image composition (pubic symphysis, urethra in anterior and perineum, levator ani in posterior compartments). The pressure gradient distributions $(\mathrm{kPa} / \mathrm{mm})$ for vaginal wall deformation in the orthogonal to vaginal canal direction (up and down) reveal elasticity distribution along the vagina.

Comparative Test 2 (probe elevation) results for patients with normal pelvic support (no POP) and with stage II POP are presented in Figure 3. Tissue deformation (up to $45 \mathrm{~mm}$ ) in posterior compartment (see Figure 3(C)) allows acquisition of pressure feedback patterns versus the probe angle to quantify the pelvic floor support at Levels I-III, as defined by DeLancey [19], because the deeper support structures contribute to the pressure patterns on the vaginal wall at such deformations [10]. The tactile ultrasound image fusion (see Figure 4(A) and Figure 4(B)) provides anatomical identification of the levels of support.

Test 3 (probe rotation by 360 degrees) allows acquisition of circumferential tactile and ultrasound images, which can be transformed into a 3D ultrasound image of the pelvic floor structures around the vagina. The angled tactile patterns from vaginal walls can be placed inside that 3D ultrasound image (see yellow pressure line in Figure 4(A)) to analyze suspicious findings with increased pressure feedback. Figure 4 presents an example of such images (orthogonal cross sections) with imposed pressure patterns. Mutual orthogonal positions for ultrasound images in Figure 4(A) and Figure 4(B) are marked by green lines. Asymmetry in the circumferential pressure distribution relative the vertical dashed line (see Figure 4(A)) reveals a nodule on the left side and levator ani defect on the right upper side; this patient has stage II anterior prolapse.

Test 4 (Valsalva maneuver) results for anterior compartment are depicted in Figure 5. The left panel (Figure 5(A)) shows the anterior sagittal anatomy with pressure pattern on the vaginal wall at rest; the right panel (Figure 5(B)) shows the anterior sagittal anatomy with pressure pattern on the vaginal wall at Valsalva. In this specific case, one may see 1) unusual urethral complex (elongated, heterogeneous), 2) significant reduction in the distance (see L1) between the bladder and body surface (skin) at Valsalva, 3) urethral pressure changes from 7 $\mathrm{kPa}$ at rest to $19 \mathrm{kPa}$ at Valsalva (see maximum pressure values in Figure 5(A) and Figure 5(B)), 4) significant urethral mobility by about $13 \mathrm{~mm}$ at Valsalva (see maximum pressure shift in Figure 5(B) relative to Figure 5(A)), and 5) a bladder defect discovered in Figure 5(B).

Test 5 (voluntary muscle contraction, anterior versus posterior) results for posterior compartment are shown in Figure 6. The left panel (Figure 6(A)) shows the posterior sagittal anterior anatomy with pressure pattern on the vaginal wall at rest; the right panel (Figure 6(B)) shows the posterior sagittal anatomy with pressure pattern on the vaginal wall at muscle contraction. In this specific case, one may see 1) anorectal complex (angled to the vaginal wall), and 2) three pressure peaks at muscle contraction. It allows characterization of the contractile strength of the pelvic floor muscles from the tactile data and their 

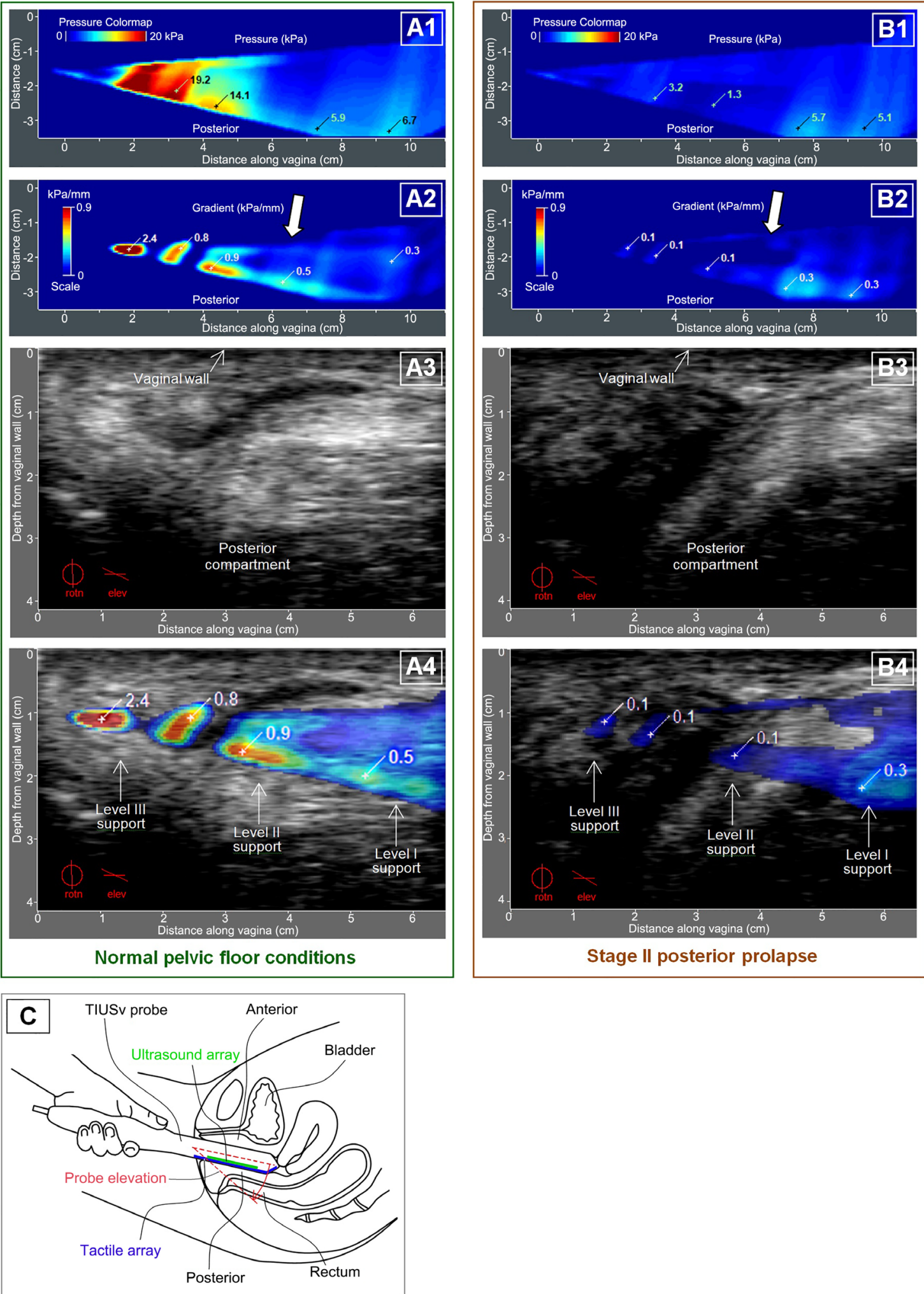

Figure 3. Normal (left, A1 - A4) and POP Stage II (right, B1 - B4) posterior conditions imaged by TIUSv at probe elevation against posterior compartment (Test 2 results). (A1, B1) tactile images (pressure patterns); (A2, B2) gradient images calculated from the tactile images A1 and B1; (A3, B3) ultrasound images; (A4, B4) tactile and ultrasound image fusion allows assessment of pelvic floor support; (C) sagittal diagram of the pelvic floor at probe elevation versus posterior compartment. 

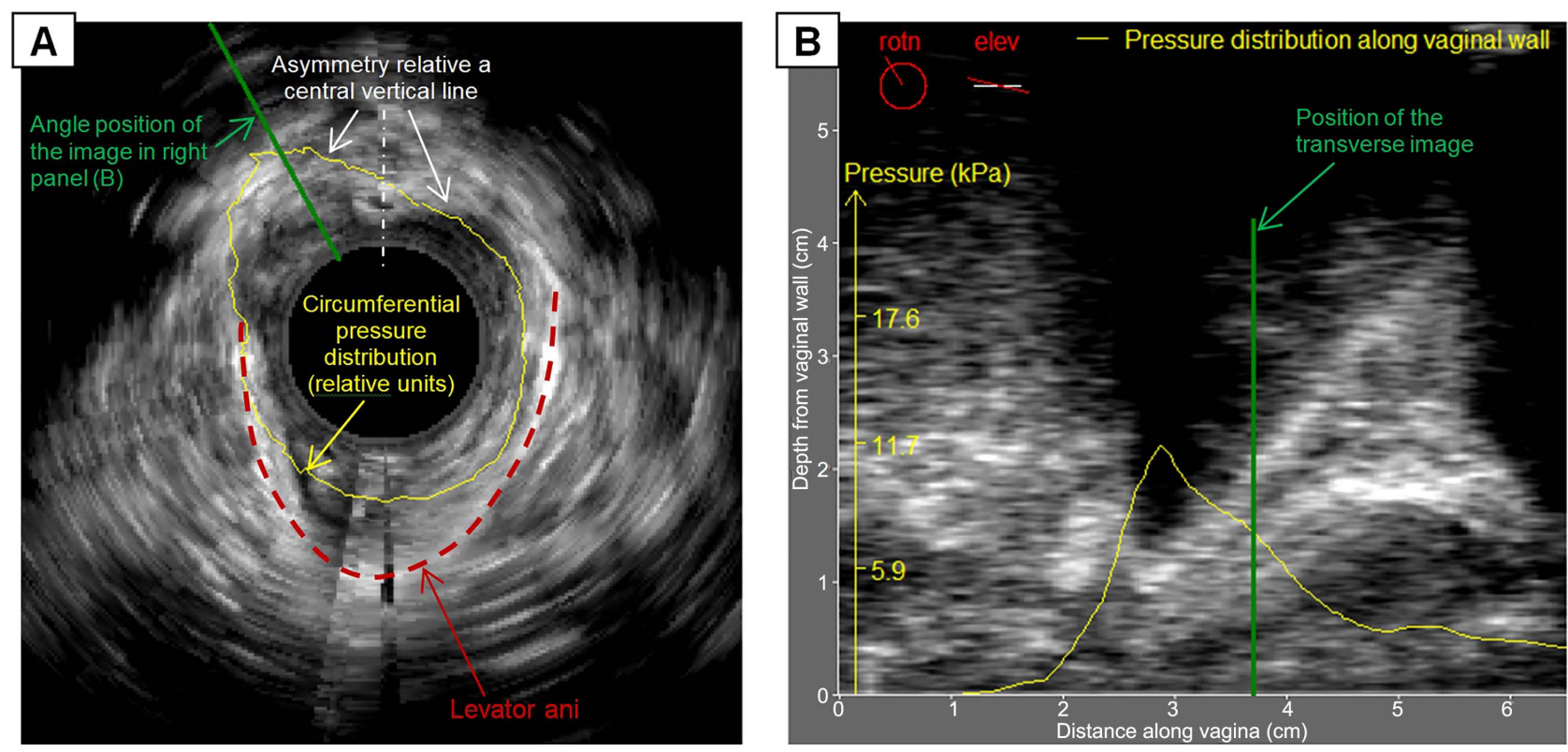

Figure 4. Orthogonal ultrasound images with pressure distribution patterns in Test 3 (probe rotation). (A) transverse image; (B) image along the vagina at specified angle for 53 y.o. patient with stage II anterior prolapse.
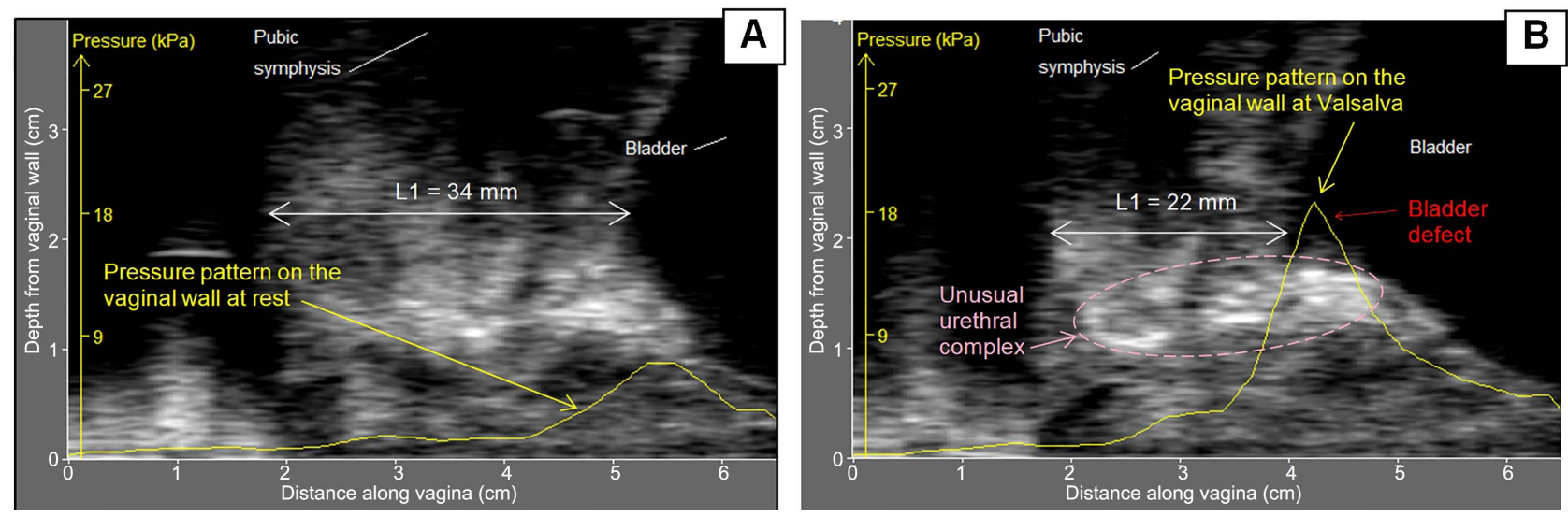

Figure 5. Anterior sagittal images of a patient with anterior Stage III POP and overactive bladder at rest (A) and Valsalva maneuver in Test 4 (B).

identification from the ultrasound images. Specifically, puboperineal, puborectal and pubovaginal muscles were identified as responsible for the peaks at the muscle contraction observed in Figure 6(B). Contractive pressure values for these muscles can be considered as biomechanical parameters (markers) characterizing pelvic function.

Test 6 (voluntary muscle contraction, left versus right sides) results for the left side are shown in Figure 7. It allows characterization of the contractile strength of the pelvic floor muscles from the tactile data and their identification from the ultrasound images. Specifically, Puboperineal and puborectal muscles were identified as being responsible for the two peaks at the muscle contraction observed in Figure 7(A)). Contractive pressure values for these muscles can be considered as biomechanical parameters (markers) characterizing pelvic function. 

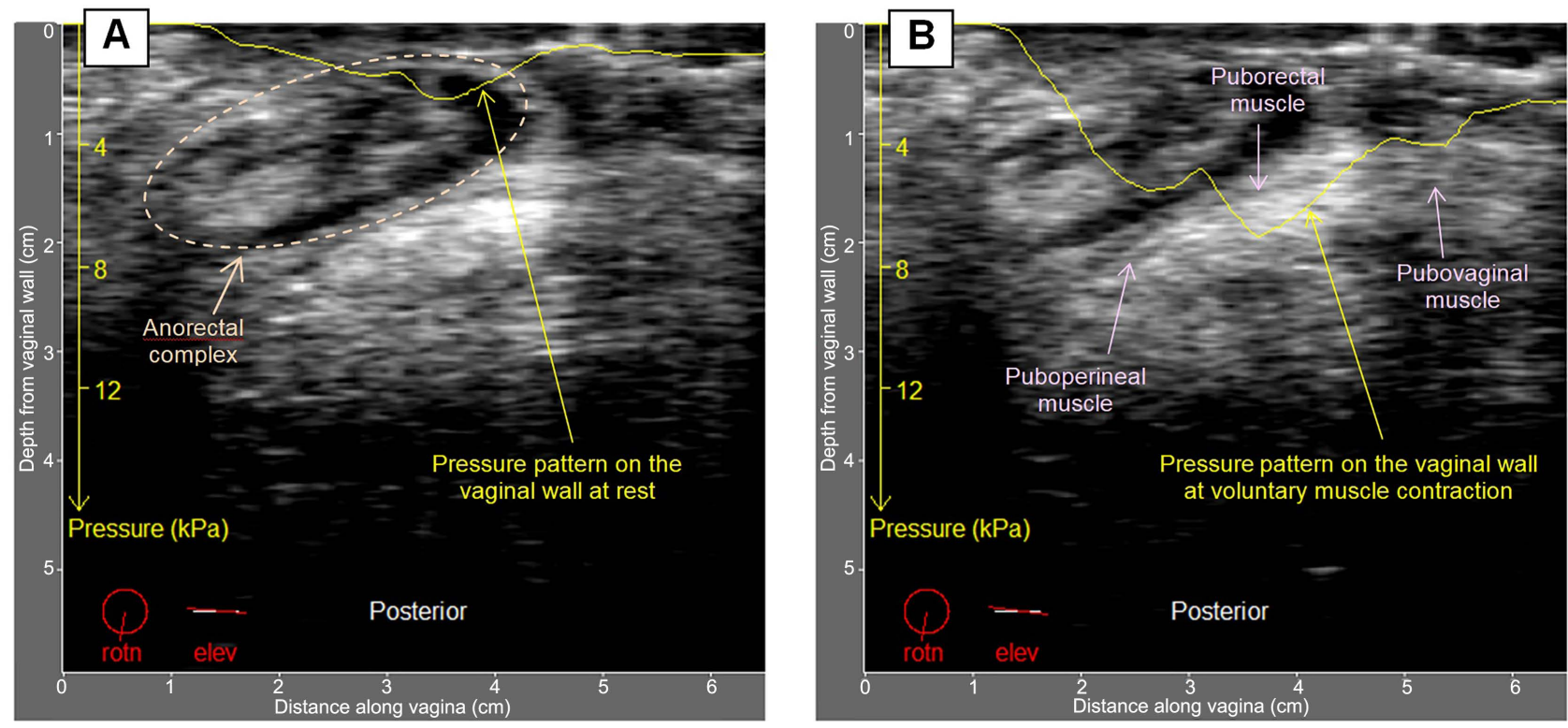

Figure 6. Posterior images of a patient without posterior prolapse, but with uterine Stage II POP at rest (A) and at voluntary pelvic muscle contraction in Test 5 (B).
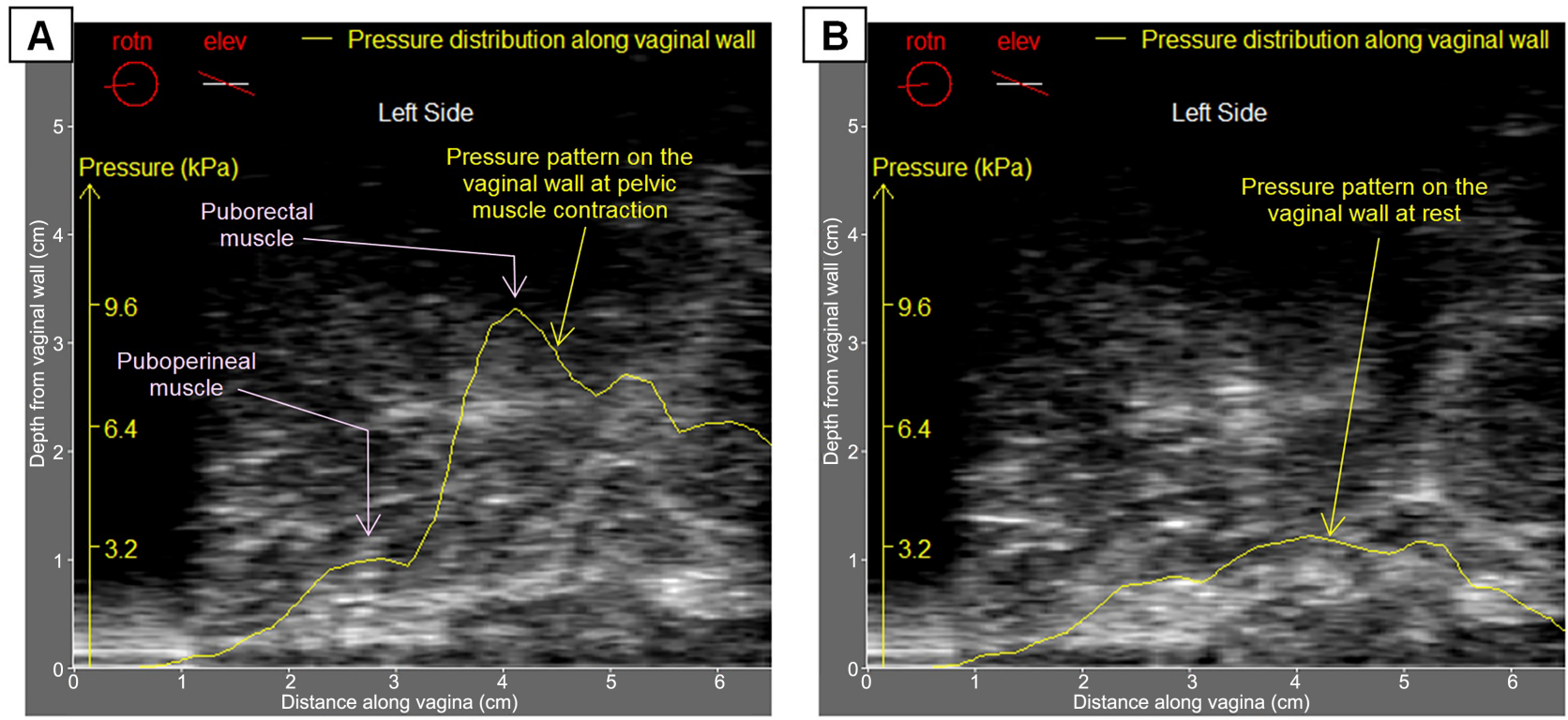

Figure 7. Left side images of a 27 y.o. patient without prolapseat rest (A) and at voluntary pelvic muscle contraction in Test 6 (B).

Test 7 provides data for involuntary pelvic muscle relaxation, when a patient is asked to squeeze and keep the strength. The pelvic muscle is relaxed involuntary to decreased pressure values. The speed of muscle relaxation for a posterior pressure peak (see Figure 8(A))) can be quantified from the tactile data in $\mathrm{kPa} / \mathrm{s}$ or $\% / s$ units. Figure $8(\mathrm{~B})$ presents the dynamic pressure profile along the posterior vaginal wall at the involuntary muscle relaxation. The relaxation speed is related to the muscle innervation state [20].

Test 8 (reflex muscle contraction on coughing) results are shown in Figure 8. An anterior ultrasound image with imposed peak pressure pattern at contraction 


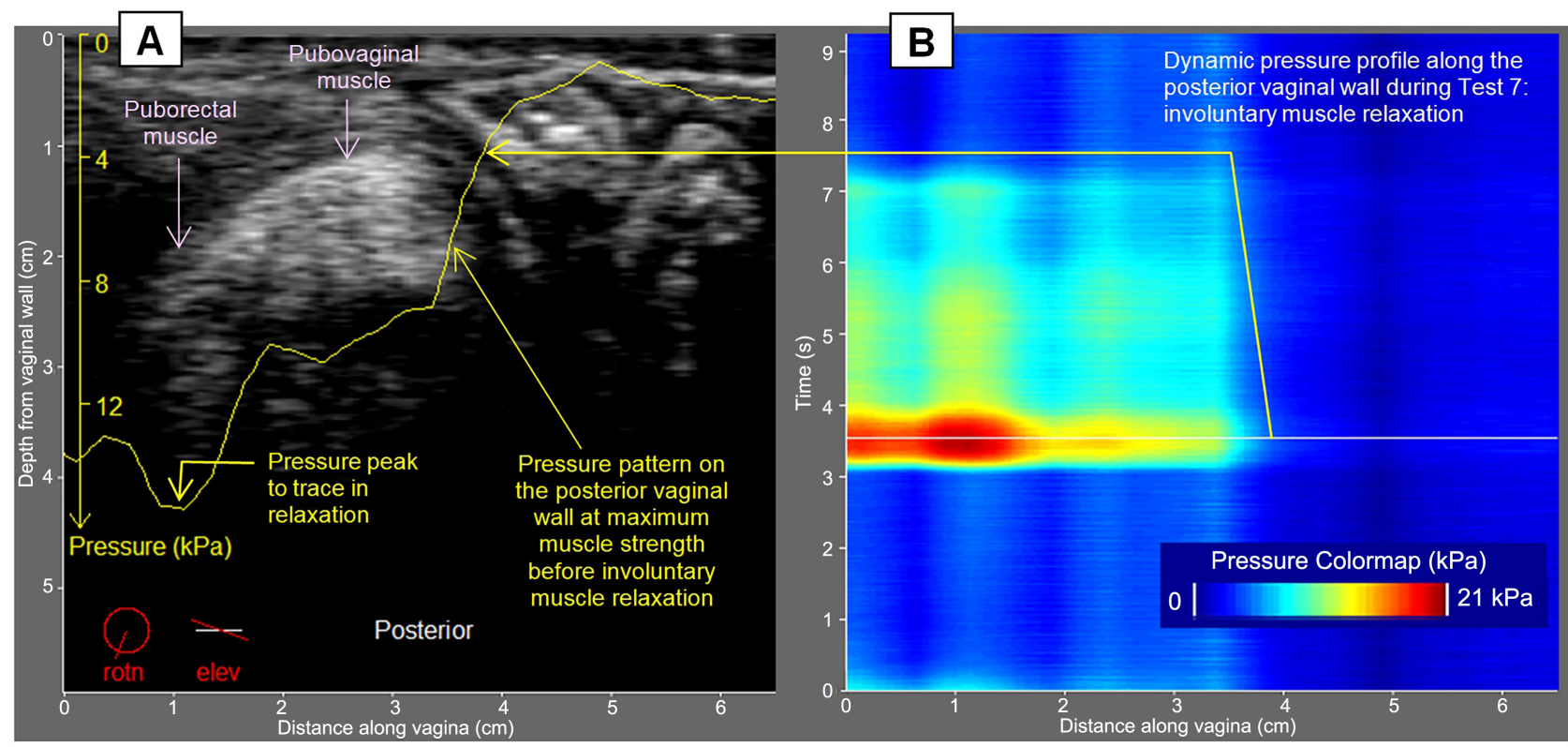

Figure 8. Images for involuntary muscle relaxation (Test 7), 37 y.o. patient without prolapse. (A) ultrasound image at maximum muscle strength, and (B) dynamic tactile image (pressure distribution on the vaginal wall as function of time - vertical axis).

is presented in Figure 9(A)); urethral complex is easy identifiable there. Figure 9(B) presents the dynamic pressure profile along the anterior vaginal wall at the reflex muscle contraction. Urethral mobility can be quantified from the tactile data in Figure 9(B). That is another biomechanical parameter (marker) characterizing pelvic function.

No TIUSv-related adverse events were reported from the participating clinical sites.

\section{Discussion}

Many pelvic floor disorders, including POP/UI, are manifested by concurrent changes in the mechanical properties of pelvic structures. Therefore, the biomechanical mapping of response to applied pressure or load within the pelvic floor and muscle contractive patterns opens new possibilities for the assessment and monitoring of the female pelvic floor conditions. There are no other physical characteristics of soft tissues with such great sensitivity to pathological and structural changes (at a micro level) than Young's modulus and shear modulus of the tissue. Detection of a mechanical heterogeneity by manual palpation is based on sensing the variations of these tissue characteristics; for instance, Young's modulus for different soft tissues varies over four orders of magnitude and, even within one tissue, may change by many orders of magnitude in diseased conditions [21]. Further, the functional imaging of active pelvic structures with their contractive force/pressure measurements seems highly important for the characterization of the female pelvic floor conditions and diagnosis.

The TIUSv delivers a new functional imaging of the pelvic floor active structures (muscles) contributing to pelvic floor disorders. This possibility arises from 


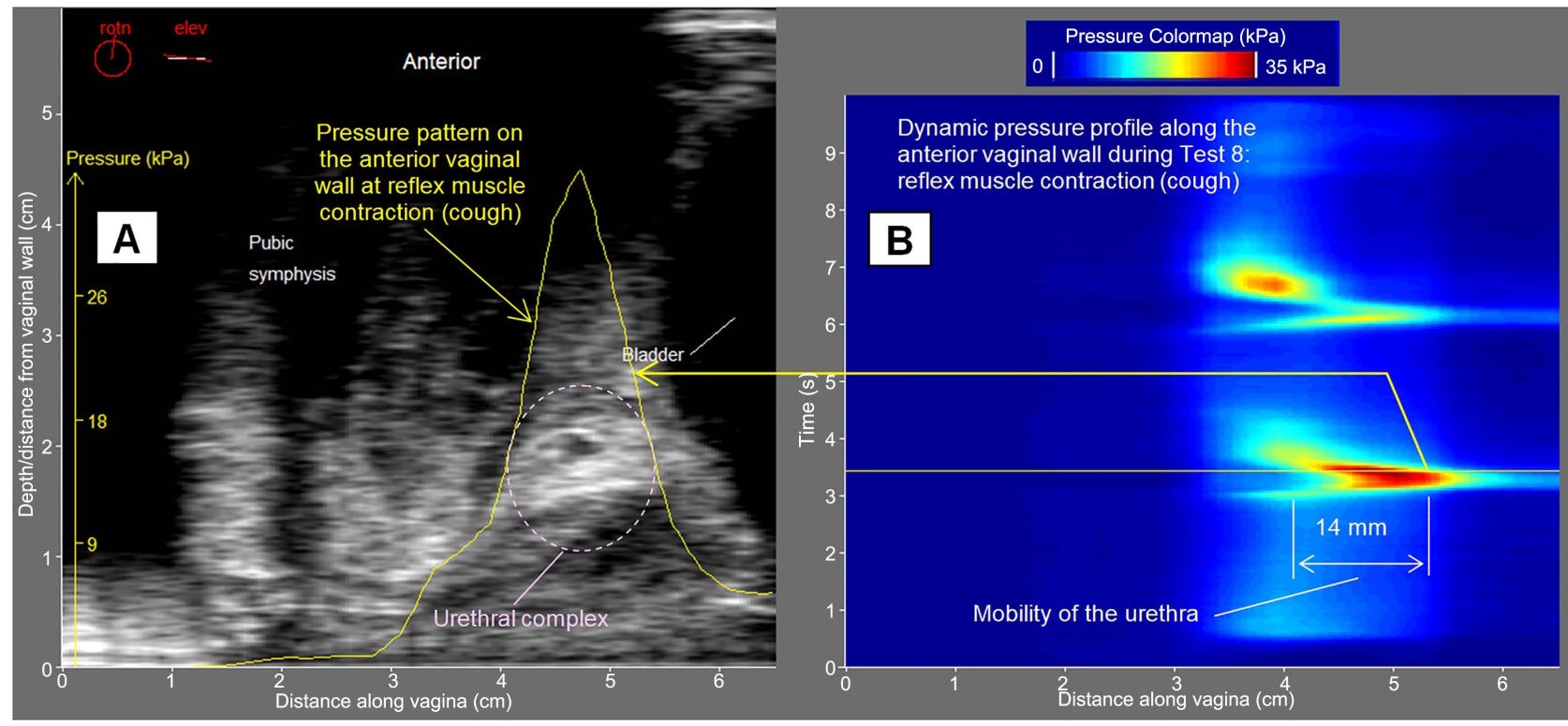

Figure 9. Images for involuntary muscle contraction (cough, Test 8). (A) ultrasound image clearly demonstrates that the strong pressure signal (yellow graph) is coming from the urethra, not from pubic symphysis, for 44 y.o. patient with normal pelvic support (no POP), but with SUI and hyperactive urethra; and (B) dynamic tactile image (pressure distribution on the vaginal wall as function of time - vertical axis) allows measurement of urethral mobility.

the fusion of tactile and ultrasound techniques. Such fusion has fundamental importance, because these technologies are complementary to each other: tactile images provide stress data and ultrasound images provide strain data as well as anatomy for the same tissue during its deformation. This fusion allows elasticity characterization as a stress-to-strain ratio, according to its classical definition [22].

To the best of our knowledge, no one has ever attempted to quantify soft tissue elasticity, pelvic support and function with the fusion of tactile and ultrasound transducers in one probe. In the proposed approach, the tactile sensor arrays provide the tissue stress data, while the ultrasound transducers provide anatomical and the tissue strain data. Figures 2-9 show examples of the image/data fusion for the studied patients with TIUSv probe. Both modalities (tactile and ultrasound) have imaging resolution sufficient for recognition and segmentation of the basic pelvic floor components.

The TIUSv probe has the same design (geometrical sizes) as the Vaginal Tactile Imaging (VTI) probe (oval $16 \mathrm{~mm} \times 31 \mathrm{~mm}$ ). In earlier studies, $54 \%$ of the patients classified VTI comfort level as more comfortable than manual palpation, $36 \%$ as the same, and $10 \%$ as less comfortable than manual palpation [23].

Computational procedures with only tactile data for Tests $1-8$ generate 52 biomechanical parameters (12 related to tissue elasticity, 12 to pelvic support and 28 to pelvic function) [24]. Tactile and ultrasound data can provide calculation of 33 new biomechanical parameters (not available in the VTI); among them, 8 use only ultrasound data (Minimal levator hiatus area, Urethral length, Levator plate descent angle, Anorectal angle, Perineal muscle resting position, 
Perineal muscle squeeze position, Vaginal levator resting position, Vaginal levator squeeze position [14]), and 25 parameters use both tactile and ultrasound data additionally characterizing tissue elasticity, pelvic support and function. A finite element model (FEM) of the pelvic floor to simulate tissue deformations in anterior and posterior compartments by the TIUSv probe will provide more accurate tissue elasticity (Young's modulus, $\mathrm{kPa}$ ) calculation based on tactile (stress) and ultrasound (strain) data acquired in tests 1 - 3. Figure 2 illustrates the concept of tactile/ultrasound imaging, where the pressure spatial gradient $(\mathrm{kPa} / \mathrm{mm})$ characterizes tissue elasticity. The FEM development is beyond the current research. Recently, we have designed the female pelvic floor FEM for tissue deformation in the posterior compartment for obstetrical application [25].

The American Urogynecologic Society conducted a survey among the leaders in the field, including clinicians, clinical and basic science researchers, and representatives from government agencies, industry, patient advocacy groups, and the public. The objective was to identify critical areas of need for future research. The survey identified mechanistic research on pelvic supportive structures (1st position), clinical research to optimize outcomes after POP surgery (4th position) and evidence-based quality measures for POP outcomes (5th position) as the main needs [26]. Further, this panel stated, "For POP, there is a need to describe interdependence of load-bearing structures such as levator ani muscles, connective tissue, and nerves because these structures relate to the bony anatomy and to the proposed components of pelvic support. Furthermore, there is a need to understand how expulsive forces are applied and distributed." The proposed approach with the TIUSv seems relevant to these objectives.

As abnormalities of the different pelvic compartments are often interrelated, their thorough diagnostic characterization is paramount for proper disease management and treatment [27]. Hence, the female pelvic floor assessment and diagnostics must include dynamic stress/strain and functional imaging to facilitate the identification and characterization of the pelvic floor structures contributing to POP/UI development. The fusion of tactile (stress, surface strain) and ultrasound (anatomical, 2D strain) images promises to provide such a possibility. Specifically, this fusion may provide (not available currently in clinical practice) the following:

1) Biomechanical characterization of levator ani muscles (pubovaginal, puboanal, puborectal, iliococcygeal), perineum, urethra and key ligaments (cardinal and uterosacral) critical in POP/UI development;

2) A $3 D$ imaging of the anatomic and mechanical defects within the female pelvic floor;

3) Anatomical and functional information necessary for a custom pessary design;

4) 3D biomechanical data for computer simulation of surgical procedures and treatment effectiveness;

5) The objective and quantitative pre- and post-treatment assessment of pelvic 
floor conditions to improve evidence-based management in urogynecology [10].

The strength of this study is that it is the first clinical exploration and demonstration of the new possibilities in biomechanical characterization of the female pelvic floor. The discovery of novel biomechanical markers for critical soft tissues/structures may provide extended scientific knowledge and improve clinical practice. One of the key tasks of the TIUSv is to assist the clinicians in differentiating normal soft tissue with an expected elasticity range from affected tissues with distinctive biomechanical properties. The fusion of tactile and ultrasound images and tissue/structure characterization may improve the diagnostic accuracy of complex pelvic floor disorders and selection of an optimal treatment. Biomechanical mapping of the female pelvic floor before the POP surgery and probability distributions for success/failure of specific surgical procedures and their combinations to recover the biomechanical status of the pelvic floor may change clinical practice [28].

The weakness of this study is the limited sample size studied, which does not allow deriving any dependencies of the biomechanical parameters versus the pelvic conditions. That will be the subject of future research. Another weakness is image dependence on operator's skill level. In general, an examination with a tactile and/or ultrasound imaging probe is operator-dependent and requires appropriate training.

\section{Conclusion}

Vaginal tactile and ultrasound image fusion provides unique data for biomechanical characterization of the female pelvic floor. Bringing novel biomechanical characterization for critical soft tissues/structures may provide extended scientific knowledge and improve clinical practice.

\section{Acknowledgements}

Research reported in this publication was supported by research grant R43HD09805 from Eunice Kennedy Shriver National Institute of Child Health \& Human Development (NICHD). The content is solely the responsibility of the authors and does not necessarily represent the official views of the National Institutes of Health.

\section{Conflicts of Interest}

The authors declare no conflicts of interest regarding the publication of this paper. Egorov is a CEO and a minor shareholder of Advanced Tactile Imaging, Inc. Egorov has submitted a patent application related to the reported approach. Raalte is a minor shareholder of Advanced Tactile Imaging, Inc. Shobeiri reports no conflict of interest.

\section{References}

[1] Iglesia, C.B. and Smithling, K.R. (2017) Pelvic Organ Prolapse. American Family Phy- 
sician, 96, 179-185.

[2] Weintraub, A.Y., Glinter, H. and Marcus-Braun, N. (2020) Narrative Review of the Epidemiology, Diagnosis and Pathophysiology of Pelvic Organ Prolapse. International Brazilian Journal of Urology, 46, 5-14. https://doi.org/10.1590/s1677-5538.ibju.2018.0581

[3] Al-Mukhtar Othman, J., Åkervall, S., Milsom, I. and Gyhagen, M. (2017) Urinary Incontinence in Nulliparous Women Aged 25-64 Years: A National Survey. The American Journal of Obstetrics and Gynecology, 216, 149.e1-149.e11.

https://doi.org/10.1016/j.ajog.2016.09.104

[4] Kieserman-Shmokler, C., Swenson, C.W., Chen, L., Desmond, L.M., Ashton-Miller, J.A. and DeLancey, J.O. (2020) From Molecular to Macro: The Key Role of the Apical Ligaments in Uterovaginal Support. The American Journal of Obstetrics and Gynecology, 222, 427-436. https://doi.org/10.1016/j.ajog.2019.10.006

[5] Shobeiri, S. (2017) 2D/3D Endovaginal and Endoanal Instrumentation and Techniques. In: Practical Pelvic Floor Ultrasonography: A Multi-Compartmental Approach to $2 D / 3 D / 4 D$ Ultrasonography of Pelvic Floor, Springer-Verlag, New York, 19-44. https://doi.org/10.1007/978-1-4614-8426-4 2

[6] Petros, P. (2010) The Female Pelvic Floor: Function, Dysfunction and Management According to the Integral Theory. Springer Verlag, Berlin, 1-330.

[7] Wu, J.M., Matthews, C.A., Conover, M.M., Pate, V. and Jonsson Funk, M. (2014) Lifetime Risk of Stress Urinary Incontinence or Pelvic Organ Prolapse Surgery. $O b$ stetrics \& Gynecology, 123, 1201-1206. https://doi.org/10.1097/AOG.0000000000000286

[8] Smith, F.J., Holman, C.D.A.J., Moorin, R.E. and Tsokos, N. (2010) Lifetime Risk of Undergoing Surgery for Pelvic Organ Prolapse. Obstetrics \& Gynecology, 116, 10961100. https://doi.org/10.1097/AOG.0b013e3181f73729

[9] Jelovsek, J.E., Barber, M.D., Norton, P., Brubaker, L., Gantz, M., Richter, H.E., et al. (2018) Effect of Uterosacral Ligament Suspension vs Sacrospinous Ligament Fixation with or without Perioperative Behavioral Therapy for Pelvic Organ Vaginal Prolapse on Surgical Outcomes and Prolapse Symptoms at 5 Years in the Optimal Randomized Clinical Trial. JAMA, 319, 1554-1565. https://doi.org/10.1001/jama.2018.2827

[10] Lucente, V., van Raalte, H., Murphy, M. and Egorov, V. (2017) Biomechanical Paradigm and Interpretation of Female Pelvic Floor Conditions before a Treatment. International Journal of Women's Health, 9, 521-550. https://doi.org/10.2147/IJWH.S136989

[11] Dietz, H.P. (2012) Female Pelvic Floor Dysfunctiong-An Imaging Perspective. Nature Reviews Gastroenterology \& Hepatology, 9, 113-121. https://doi.org/10.1038/nrgastro.2011.213

[12] Farouk El Sayed, R. (2013) The Urogynecological Side of Pelvic Floor MRI: The Clinician's Needs and the Radiologist's Role. Abdominal Imaging, 38, 912-929. https://doi.org/10.1007/s00261-012-9905-3

[13] Bahrami, S., Khatri, G., Sheridan, A.D., Palmer, S.L., Lockhart, M.E., Arif-Tiwari, H., et al. (2019) Pelvic Floor Ultrasound: When, Why, and How? Abdominal Radiology. https://doi.org/10.1007/s00261-019-02216-8

[14] Shobeiri, S. (2017) Pelvic Floor Anatomy. Practical Pelvic Floor Ultrasonography: A Multicompartmental Approach to 2D/3D/4D Ultrasonography of the Pelvic Floor. Second Edition, Springer International Publishing, New York, 1-368. https://doi.org/10.1007/978-3-319-52929-5 1 
[15] Dietz, H.P. (2019) Ultrasound in the Assessment of Pelvic Organ Prolapse. Best Practice \& Research: Clinical Obstetrics \& Gynaecology, 54, 12-30. https://doi.org/10.1016/j.bpobgyn.2018.06.006

[16] Egorov, V., Shobeiri, S., Takacs, P., Hoyte, L., Lucente, V. and van Raalte, H. (2018) Biomechanical Mapping of the Female Pelvic Floor: Prolapse versus Normal Conditions. Open Journal of Obstetrics and Gynecology, 8, 900-924. https://doi.org/10.4236/ojog.2018.810093

[17] Sarvazyan, A., et al. (2011) An Overview of Elastography-An Emerging Branch of Medical Imaging. Current Medical Imaging Reviews, 7, 255-282. https://doi.org/10.2174/157340511798038684

[18] Bump, R.C., Mattiasson, A., Bo, K., Brubaker, L., DeLancey, J.O.L., Klarskov, P., et al. (1996) The Standardization of Terminology of Female Pelvic Organ Prolapse and Pelvic Floor Dysfunction. The American Journal of Obstetrics and Gynecology, 175, 10-17. https://doi.org/10.1016/S0002-9378(96)70243-0

[19] DeLancey, J.O.L. (2016) Pelvic Floor Anatomy and Pathology. In: Biomechanics of the Female Pelvic Floor, Elsevier, Amsterdam, 13-51.

https://doi.org/10.1016/B978-0-12-803228-2.00002-7

[20] Cardenas-Trowers, O., Meyer, I., Markland, A.D., Richter, H.E. and Addis, I. (2018) A Review of Phytoestrogens and Their Association with Pelvic Floor Conditions. Female Pelvic Medicine \& Reconstructive Surgery, 24, 193-202. https://doi.org/10.1097/SPV.0000000000000559

[21] Sarvazyan, A. (2001) Elastic Properties of Soft Tissues. In: Levy, M., Bass, H. and Stern, R.R., Eds., Handbook of Elastic Properties of Solids, Liquids and Gases, Vol. 3, Academic Press, New York, 107-127. https://doi.org/10.1016/B978-012445760-7/50057-5

[22] Timoshenko, S. and Goodier, J. (1951) Theory of Elasticity. McGraw-Hill Book Company, New York, 506 p.

[23] van Raalte, H. and Egorov, V. (2015) Tactile Imaging Markers to Characterize Female Pelvic Floor Conditions. Open Journal of Obstetrics and Gynecology, 5, 505 515. https://doi.org/10.4236/ojog.2015.59073

[24] Egorov, V., Lucente, V., Shobeiri, S., Takacs, P., Hoyte, L. and van Raalte, H. (2018) Biomechanical Mapping of the Female Pelvic Floor: Uterine Prolapse versus Normal Conditions. EC Gynaecology, 7, 431-446.

https://doi.org/10.4236/ojog.2018.810093

[25] Rusavy, Z., Kalis, V., Aglyamov, S. and Egorov, V. (2020) Feasibility and Safety of Antepartum Tactile Imaging. International Urogynecology Journal, 1-7. https://doi.org/10.1007/s00192-020-04552-6

[26] Siddiqui, N.Y., Gregory, W.T., Handa, V.L., Delancey, J.O.L., Richter, H.E., Moalli, P., et al. (2018) American Urogynecologic Society Prolapse Consensus Conference Summary Report. Female Pelvic Medicine \& Reconstructive Surgery, 24, 260-263. https://doi.org/10.1097/SPV.0000000000000533

[27] Bø, K. and Sherburn, M. (2005) Evaluation of Female Pelvic-Floor Muscle Function and Strength. Physical Therapy, 85, 269-282. https://doi.org/10.1093/ptj/85.3.269

[28] Egorov, V., Takacs, P., Shobeiri, S.A., Hoyte, L., Lucente, V., van Raalte, H., et al. (2021) Predictive Value of Biomechanical Mapping for Pelvic Organ Prolapse Surgery. Female Pelvic Medicine \& Reconstructive Surgery, 27, e28-e38. https://doi.org/10.1097/SPV.0000000000000786 